\title{
The Cricket and the Ant: \\ Organizational Tradeoffs in Changing Environments
}

\author{
Gábor Péli*
}

SOM-theme B: Marketing and interaction between firms

\begin{abstract}
Organizations face tradeoffs when they adopt strategies in changing resource environments. The type of tradeoff depends on the type of resource change. The paper gives an organizational tradeoff model for quantitative change. I call that the "Cricket and Ant" (CA), because the pertaining strategies resemble to the cricket and ant behavior in La Fontaine's famous fable. I demonstrate that organizational ecology's niche theory and $r$ - $K$-strategist theory obtain as special cases of CA; their predictions on selection derive as theorems, if their respective boundary conditions are represented in the formal model. New selection conclusions also derive, prone to empirical testing.
\end{abstract}

* Thank you Glenn Carroll, Michael Hannan, Jaap Kamps and László Pólos for your comments on a previous version of the paper. 


\section{Environmental Change}

Organizational survival, success, dominance depend on the match between organizational strategy and the pattern of environmental change. When choosing a strategy, organizations may face tradeoffs. For example, in the niche theory of organizational ecology (OE), generalist organizations adapt to more than one environmental conditions, while specialists adapt only to one in which they excel (Freeman and Hannan 1983). In the so called $r$-and $K$-strategy tradeoff of $\mathrm{OE}$, the $K$-strategist organizations invest in skill development that enables them to survive in tightly packed markets (efficient producers), while the easy-toassemble, first mover $r$-strategists proliferate rapidly under conditions of resource abundance (Hannan and Freeman 1989). Organizations may also buffer out environmental fluctuations by accumulating reserves (Thompson 1967; Kamps and Pólos 1999). Then, the price of buffering comes again in the form of damped growth in membership at the level of the organizational population. Adopting a strategy has long lasting consequences for the organization, because strategy change requires fundamental reorganization hindered by structural inertia (Hannan and Freeman 1984). Moreover, it is hard to foresee the pattern of environmental change, and this makes the rationality of basic strategy change dubious. Therefore, organizations that keep their original strategy will be strongly represented among the long term survivors (Péli, Pólos and Hannan 2000).

The environment in $\mathrm{OE}$ is characterized by the configuration of resources that the populating organizations might exploit. A resource configurations can be seen as a vector in which components tell the respective availabilities of the different resource sorts. Note that it is the resource availability that organizations perceive and not the externally given absolute stock or inflow of resources. Externally given environmental resource input and the level of resource consuption jointly determine resource availability.

Both GS and RK focuses on the temporal change of resources (assuming tacitly that spatial inhomogeneities play no considerable role). The paper goes along with this characterization. I adopt the typology of Hannan and Freeman on environmental change with three variables that describe abrupt environmental change(1977, 1989). Change separates time periods of homogeneous resource conditions called environmental patches. A temporal sequence composed of the old and the subsequent new patch gives the pattern of environmental change.

The three dichotomic variables that determine the pattern of environmental change are dissimilarity, variability and grain (Figure 1). 


\section{Figure 1. Patterns of environmental change}

High Variability

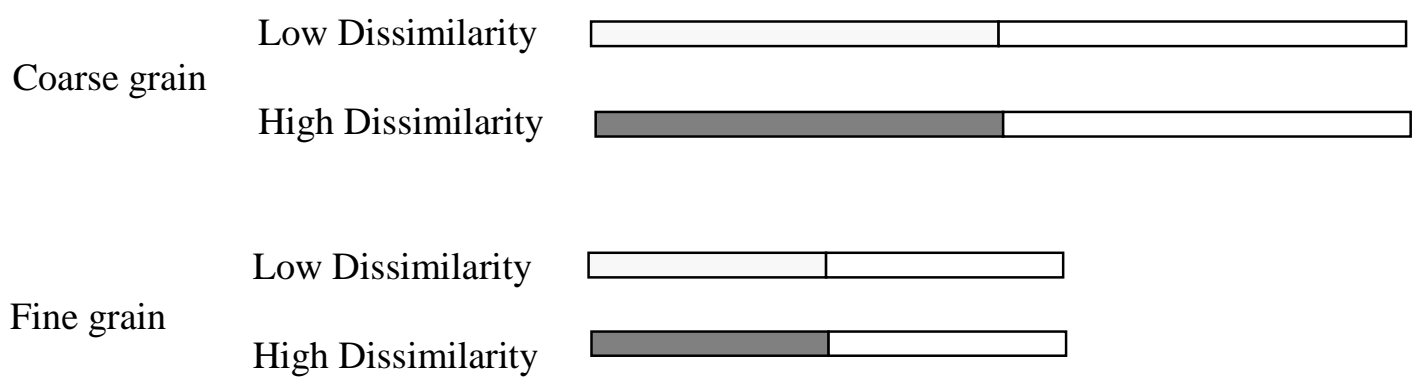

Low Variability

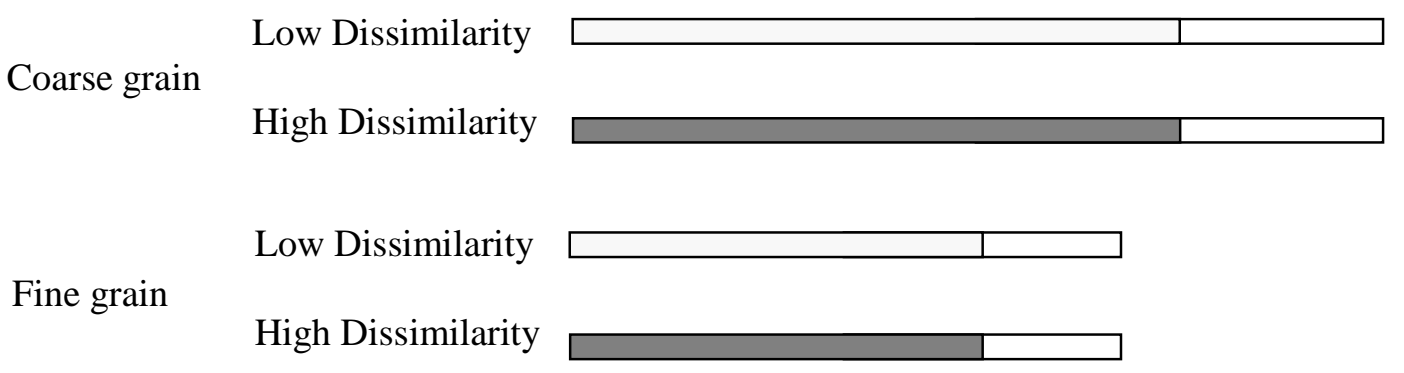

The blocks stand for the temporal patches for which a certain environmental state holds. Environmental dissimilarity is visualized as tint difference between blocks. 
Environmental dissimilarity tells the degree of difference between the old and the new resource conditions. The bigger this difference, the more difficult to cope with both environmental conditions. Environmental variability is about the relative duration of the old and the new patches; it tells if one of the environmental conditions holds most of the time or not. If the two patches are about the same duration, then variability is high. If one of the patches is much shorter that the other, than it represents only a transient period of change that is relatively easy to buffer out; then, environmental variability is low. Environmental grain is about the absolute length of the sequence composed of two subsequent patches. In case of coarse grain, this sequence is long, so the average patch length is big. In case of fine grain, patches are short.

The paper adresses a further aspect of environmental resource change. Is it qualitative or quantitative? Is it the composition or the amount of resource that modifies in the environment?

First, resource change can be qualitative. Then, it is not the amount but the composition of resources that modifies. There may be environmental resources available all the time, but different resources, and organizations that can absorb one setting may not be able to utilize another. For example, customers' demand is a key resource for organizations (Carroll 1985). A radical change in customer taste can eliminate organizations that specialized to a taste that becomes soon obsolete.

Second, the change in resource conditions can be quantitative. Then, it is the presence and the lack of available resource that characterize the two environmental conditions. The amount of unbound resource may decrease due to endogenous processes in the market. As the population builds up, organizations finally absorb all free resource, that is the carrying capacity of the market is met. The environmental resource flow may be the same, but there is no unbound resource anymore, and from that point, organizations face head-on competition. Think of the saturation of a brand new market, or of the appearance of a radically new version of an existing product due to technological change.

In a third case, resource conditions still change quantitatively, but the change is exogenous, like seasonal shifts. Then, it is not the stock of unbound resource but the absolute amount of environmental resource offer that changes abruptly. Examples are expansions and contractions in purchasing power, or summer and winter seasons in a holiday resort. The three cases can mix. But for the sake of analytical clarity, I address them separately.

\section{Organizational Tradeoffs}

The three modes of resource change mentioned above correspond to three tradeoffs in organizational strategies; two have been thoroughly studied in OE, the third is new. 
The first case, qualitative change, characterizes OE's niche theory (Freeman and Hannan 1983, Hannan and Freeman 1989). Here, the tradeoff is between organizational niche breadth and the quality of performance (principle of allocation). Generalist organizations can cope with qualitatively different resource environments. But adaptation to different environments, that is, maintaining a broad niche, involves the jack-of-all-trade dilemma: generalists do reasonably well in all environments but they excel in none. Specialists bet on one environment and perform there excellently. However, they face hard times when the environment changes. Péli (1997) gave a logical model and derived niche theory's main conclusions as theorems from the theory's basic assumptions; I refer to this generalistspecialist model as GS in the forthcoming. ${ }^{1}$

The second case, quantitative change in resource availability as the carrying capacity is reached, applies to OE's $r$ - and $K$-strategist dilemma (Hannan and Freeman 1989). Here, the tradeoff stands between rapid proliferation in early markets ( $r$-strategy) and the investment in the development in organizational skills that yield competitive advantage in crowded markets ( $K$-strategy). Péli and Masuch (1997) derived the theory's predictions on selection preferences from some basic model assumptions. I refer to the $r$ - and $K$-strategist model as RK.

The third case, exogenous quantitative change in environmental resource flow, has not been yet addressed by OE. Here, the tradeoff stands between rapid population growth and organizational reserve accumulation. The amount of total resource a population can absorb during the resourceful period is given. The question is the way how this amount of resource is distributed along population members. The population can grow steeply during the high season, ending up with many organizations with minimal reserves. Or the same amount of resource can be distributed along less but "well equipped" organizations that survive even lengthy low seasons on their supplies. I call this the Cricket and the Ant tradeoff, because of the resemblance to La Fontaine's famous cautionary tale. I refer to this model as CA.

\section{The Task}

We have now three ways how environmental resources change and three corresponding organizational tradeoffs. Two of them already have a logical model, one has not. The first task of the paper is to give an account of population dynamics in case of exogenous and quantitative environmental change. CA will give the selection preferences between the Cricket and the Ant strategies in all eight patterns of environmental change depicted in

\footnotetext{
${ }^{1}$ Bruggeman (1997) suggested another approach with somewhat different assumptions and conclusions.
} 
Figure 1. Adding a third logical model to the two existing is not only an analytical exercise. $\mathrm{CA}$ is the stepping stone for the second, main task of the paper that concerns the connections between the three bodies of organization theory.

The three population models strongly overlap in key notions and also in their characterization of change and strategy. This gives an opportunity to merge these models into one in a way that the new, general model gives back the three components (CA, GS and RK) as special cases. Consider the specific assumptions (boundary conditions) of each population theory, respectively, as "switch positions" on a general model. Setting the switch into GS, RK, and CA positions, the selection predictions of the pertaining component theory should obtain as outputs (theorems) of the general model. The predictions of GS and RK are given in advance. The selection preferences in CA will derive from the new model.

Logic provides the necessary rigor and the formal machinery for the task. However, the specification of a new logical model and its merger with two others involves technicalities. I rather concentrate on the sociological considerations. Therefore, the presented part of the machinery is cut back to its minimum that is necessary to demonstrate the core ideas.

The rest of the paper is organized as follows. Part 4 spells out the logical model for CA. Parts 5 and 6 show how CA converges into GS and RK, respectively, as the pertaining sociological specificities are applied to the general framework. Part 7 provides a discussion of the obtaining theoretical insights.

\section{The Cricket and The Ant: Reserve Accumulation or Population Growth?}

In the famous cautionary tale of La Fontaine, the ant spends the summer with hard work collecting food for the winter. The cricket lives for the day playing his violine during the summer, but suffers of hunger in the winter frost. Organizational populations may face a similar tradeoff. One option is rapid population growth in the short run. The other option is to adopt safety measures; then, a great part of the absorbed resource increases organizational supplies, in order to buffer against future resource scarcity. A similar kind of tradeoff may apply to whole economies. An example is the "golden egg laying hen" dilemma in the Eastern block countries during the cold war. The term refers to another cautionary tale about the foolish man who ate his gold egg laying hen. Then, the tradeoff was between extreme investments in the heavy industries and the immediate improvement of the poor post-war living standards. ${ }^{2}$

\footnotetext{
2 In fact, the radical cut-back of civil consumption served large scale war asset production (Szabó 1981).
} 
Consider two organizational populations, the Cricket and the Ant, that follow two characteristic resource utilization strategies. The task is to compare their long term performance from a selection perspective under different patterns of environmental change (Figure 1). Selection's preference is with the population that maintains a higher number of member organizations in the long run. The resource environment changes with time: there are high seasons with bountiful resource and low seasons characterized with the lack of resource. Organizations live on reserves during the low seasons; then, they either cease to operate (winter on a beach resort), or their production inreases their stocks (contraction of purchasing power in an economy). Call the resourceful and resourceless periods summer and winter, respectively. Resource absorption takes time. The amount of resource a population can bound depends on the length of the summer and also on the speed of resource absorption. This speed is affected by the social climate. A supporting, "warm" social climate corresponds to beneficial institutional and regulatory conditions that facilitate resource utilization.

Again, the absorbed wealth can be distributed in two ways over the member organizations. A population may have a lot of members with little reserves (Cricket), or it may be composed of less but better equipped organizations (Ant). Obviously, the first is the good option in eternal summers. But, organizational numbers fall dramatically when reserves are used up in winters. Note that this rule on population level resource allocation plays a similar role to the principle of allocation in GS, when organizations economize on the tradeoff between niche width and fitness (Freeman and Hannan 1983).

\section{The formal model $^{3}$}

Weather. First, I characterize the temporal patterns of environmental change. I give a set of logical statements (assumptions) on the three environmental variables: dissimilarity, variability and grain. The characterization proceeds verbally in the main text. Please check the forthcoming statements against the visual representation of environmental patterns in Figure 1. See pertaining logical formulae in Table 2. The applied denotations are explained in Table 1.

\footnotetext{
${ }^{3}$ No specific logical skills are required to follow the formal argument. For a more detailed account on logic, the reader can consult Gamut (1991) or Barwise and Etchemendy (1990).
} 
Table 1. The list of logical symbols, proper names, predicates and function symbols

\section{Logical symbols}

$\wedge$ - the "and" connective

$\checkmark$ - the "or" connective

$\neg$ - negation

$\rightarrow$ - "if, ... then", implication

$\leftrightarrow$ - "if and only if", bi-implication

$\forall$ - "for all", universal quantification

$\exists$ - "there exists", existential quantification

\section{Proper names}

C - the Cricket population

A - the Ant population

$\mathrm{S}$ - the "summer" period, the resourceful high season

$\mathrm{W}$ - the "winter" period, the resourceless low season

low, mod_low, medium, mod_high, high, very_high - scale values for population size short, medium, long - scale values for time periods

\section{Predicates}

Diss(low) / Diss(high) - the dissimilarity between the two seasons is low / high Grain(coarse) / Grain(fine) - the grain of environmental change is coarse / fine $\operatorname{Pop}(\mathrm{x})$ - object $\mathrm{x}$ is a population Selection_favors(x,y) - selection favors object $\mathrm{x}$ over object $\mathrm{y}$ $\operatorname{Var}($ low) / Var(high) - environmental variability is low / high Warm - the social climate is "warm", supportive $\mathrm{x} \approx \mathrm{y}-\mathrm{x}$ and $\mathrm{y}$ are similar $\mathrm{x}>\mathrm{y}-\mathrm{x}$ is much bigger than $\mathrm{y}$

\section{Function values}

beg $(\mathrm{t})$ - yields the beginning point of period $\mathrm{t}$

$\operatorname{dur}(\mathrm{x})$ - yields the duration of period $\mathrm{x}$

end $(\mathrm{t})$ - yields the endpoint of period $t$

$\mathrm{N}(\mathrm{x}, \mathrm{t})$ - yields the size of population $\mathrm{x}$ at time $\mathrm{t}$

$\operatorname{surv}(\mathrm{x})$ - the length of the survival period on reserves for members of population $\mathrm{x}$ 


\section{Table 2. Logical formulae on environmental change}

A1. If environmental variability is low, then summers are longer than winters.

$$
\operatorname{Var}(\text { low }) \rightarrow \operatorname{dur}(\mathrm{S})>\operatorname{dur}(\mathrm{W})
$$

A2. If environmental variability is high, then the seasons are of the same duration.

$$
\operatorname{Var}(\text { high }) \rightarrow \operatorname{dur}(\mathrm{S})=\operatorname{dur}(\mathrm{W})
$$

A3. If environmental grain is fine and variability is low, then winter is short.

Grain(fine $) \wedge \operatorname{Var}($ low $) \rightarrow \operatorname{dur}(\mathrm{W})=$ short

A4. If environmental grain is fine and variability is high, then winter is medium long.

Grain $($ fine $) \wedge \operatorname{Var}($ high $) \rightarrow \operatorname{dur}(\mathrm{W})=$ medium

A5. Summers are long if environmental grain is coarse.

Grain $($ coarse $) \rightarrow \operatorname{dur}(\mathrm{S})=$ long

A6. The social climate is warm if environmental dissimilarity is high, and it is not warm, if dissimilarity is low.

Diss(high) $\rightarrow$ Warm $\wedge$ Diss(low) $\rightarrow \neg$ Warm 
The first assumption states that low environmental variability means that one environmental patch (namely, the resourceful season) is much longer than the other (A1, Table 2). ${ }^{4}$ On the contrary, high environmental variability means that summers and winters are of similar duration (A2, Table 2).

Grain size is about the average durations of the environmental patches. The same average may reflect different length distributions. Environmental variablity has an effect on length distrubution (A1-2), therefore I handle the high and low variability cases separately. If environmental grain is fine, then average seasonal durations are not long. If furthermore the summer is much longer than the winter (i.e., variability is low), then the winter is really short (A3, Table 2). But if fine grain combines with high environmental variability, then seasons are not short: they are "medium" long (A4, Table 2). If grain is coarse, then seasonal durations are long on the average (A5, Table 2). It turns out that it is enough to state this fact for the summer period to obtain the predictions on selection preferences.

Environmental dissimilarity reflects the difference between the seasons' nurturing conditions. Winter completely lacks resource, not supporting any population growth. Then, social climate has no effect on growth. Therefore, the summer-winter dissimilarity depends only on summer's "warmth": high dissimilarity means supporting social climate, low dissimilarity means non-supporting social climate (A6, Table 2).

Population size changes in the high season. Both the Cricket and the Ant populations increase in members in the summer. The longer the summer, the bigger the population, other things being equal. The warmer the social climate, the faster the growth. Moreover, the population size depends on the adopted resource allocation strategy: Expand or accumulate? Four premises characterize the Ant and Cricket population sizes at the end of the high season. Long summers with a supporting social climate yield the biggest populations. The Cricket has very high number of members by the end of the summer, while the reserve accumulating Ant has a modestly high number of organizations (A7, Table 3). Short summers with a non-supportive social climate yield the smallest population sizes (A8, Table 3). Short summers with a supportive social climate and long summers with a non-supportive social climate allow for intermediate population sizes. Then, the Cricket population develops a high (but not very high) number of members; the Ant population reaches a medium size (A9-10, Table 3 ).

An assumption is needed to reflect the consequences of the accumulation vs. fast population growth tradeoff. The Cricket and the Ant populations have, respectively, little and big amount of reserves at the end of the summer. The size of the reserve stocks can be

\footnotetext{
${ }^{4}$ I address cases when summers are not shorter than winters, giving the populations a chance to accumulate reserves or to increase size.
} 
represented with the length of the survival period, the duration for which organizations live up their supplies. This survival period is long for the Ant, and it is short for the Cricket (A11).

Population size change in the low season. Three rules characterize population size change during the winter. The first rule is simple: populations preserve their size during the winter, until their member organizations can live on their supplies (A12, Table 3). However, if the winter is longer than the burnout period of reserves, then the population rapidly loses members as the reserves are lived up. The loss depends on the length of the period without supplies. If the buffer lasts most of the winter (the burnout period of reserves is only little shorter than the winter), then population losses are not so dramatic (A13, Table 3). However, if reserves are gone much before the winter ends, then organization numbers reach a bottom (A14, Table 3).

The last piece of information needed to derive selection preferences between the Cricket and the Ant populations is a formal characterization of selection preference itself. Assumption 15 states that selection's favor is with the population of bigger size at the end of the winter (Table 3). Please note that different selection measures may apply in different parts of OE. Empirical studies mostly use mortality rates for this purpose. When formalizing OE's niche theory, Péli (1997) used the population outflow as selection measure, that can correspond to different concrete measures in different empirical contexts: loss in members, loss in population mass, etc. Assumption 15 compares organization numbers. Population size is much more easy to observe than mass (population size weighed by the members' size). If the size distribution of members is even, then population size and mass are proportional to each other. However, it is a crucial element in CA that the same amount of mass that come from absorbed resource is distributed differently in the two populations: the Cricket maximizes members, the Ant maximizes reserves. The change of an observed variable, organization number, is influenced by an unobserved heterogeneity in organizational reserves. 


\section{Table 3. Logical formulae on population size}

A7. Warm $\wedge$ dur $(S)=$ long $\rightarrow \mathrm{N}(\mathrm{C}$,end(S) $)=$ very_high $\wedge \mathrm{N}(\mathrm{A}$,end(S) $)=\bmod$ _high

A8. $\neg$ Warm $\wedge \neg(\operatorname{dur}(S)=$ long $) \rightarrow \mathrm{N}(\mathrm{C}$,end $(S))=$ medium $\wedge \mathrm{N}($ A, end $(S))=$ mod_low

A9. $\quad$ Warm $\wedge \neg(\operatorname{dur}(S)=$ long $) \rightarrow \mathrm{N}(\mathrm{C}$,end(S) $)=$ high $\wedge \mathrm{N}(\mathrm{A}$,end(S) $)=$ medium

A10. $\neg$ Warm $\wedge$ dur $(S)=$ long $\rightarrow \mathrm{N}(C$,end $(S))=$ high $\wedge \mathrm{N}(A$, end $(S))=$ medium

A11. The survival period on reserves is short for the Cricket, and it is long for the Ant. $\operatorname{surv}(\mathrm{C})=\operatorname{short} \wedge \operatorname{surv}(\mathrm{A})=$ long

A12. Populations preserve their size if their reserves last all winter $\forall \mathrm{x}[\operatorname{Pop}(\mathrm{x}) \wedge \operatorname{surv}(\mathrm{x}) \geq \operatorname{dur}(\mathrm{W}) \rightarrow \mathrm{N}(\mathrm{x}, \operatorname{beg}(\mathrm{W}))=\mathrm{N}(\mathrm{x}, \mathrm{end}(\mathrm{W}))]$

Read: "For all $\mathrm{x}$, if $\mathrm{x}$ is a population, and the survival on reserves of $\mathrm{x}$ is longer or equal to winter's duration, then $\mathrm{x}$ has the same size at the beginning and at the end of the winter."

A13. The effects of insufficient reserves on population size in the short run.

$\forall \mathrm{x}[\operatorname{Pop}(\mathrm{x}) \wedge \operatorname{dur}(\mathrm{W})>\operatorname{surv}(\mathrm{x}) \wedge \operatorname{dur}(\mathrm{W}) \approx \operatorname{surv}(\mathrm{x}) \rightarrow$

$(\mathrm{N}(\mathrm{x}, \operatorname{beg}(\mathrm{W}))=$ very_high $\rightarrow \mathrm{N}(\mathrm{x}$, end $(\mathrm{W}))=$ high $) \wedge$

$\left(\mathrm{N}(\mathrm{x}, \operatorname{beg}(\mathrm{W}))=\right.$ high $\rightarrow \mathrm{N}(\mathrm{x}, \mathrm{end}(\mathrm{W}))=\bmod \_$high $) \wedge$

$(\mathrm{N}(\mathrm{x}$, beg $(\mathrm{W}))=$ medium $\rightarrow \mathrm{N}(\mathrm{x}$, end $(\mathrm{W}))=$ low $)]$

Read: "For all $\mathrm{x}$, if $\mathrm{x}$ is a population, and winter's duration is longer than but similar to the survival length of $x$ on reserves, then a very high number of members at winter's beginning implies highnumber at its end; high beginning number implies modestly high number at the end; a medium beginning number implies low number at the end."

A14. The effects of insufficient reserves on population size in the long run. $\forall \mathrm{x}[\mathrm{Pop}(\mathrm{x}) \wedge \operatorname{dur}(\mathrm{W}) \gg \operatorname{surv}(\mathrm{x}) \rightarrow \mathrm{N}(\mathrm{x}$, end $(\mathrm{W}))=$ low $]$

Read: "For all $\mathrm{x}$, if $\mathrm{x}$ is a population, and winter's duration is much longer than x's survival period on reserves, then $x$ has low number of members at the end of the winter."

A15. Selection favors the population that is bigger at the end. $\forall \mathrm{x}, \mathrm{y}[\operatorname{Pop}(\mathrm{x}) \wedge \operatorname{Pop}(\mathrm{y}) \wedge \mathrm{N}(\mathrm{x}, \mathrm{end}(\mathrm{W}))>\mathrm{N}(\mathrm{y}, \mathrm{end}(\mathrm{W})) \rightarrow$ Selection_favors $(\mathrm{x}, \mathrm{y})]$

Read: "For all $\mathrm{x}$ and $\mathrm{y}$, if $\mathrm{x}$ and $\mathrm{y}$ are populations, and $\mathrm{x}$ has bigger size than $\mathrm{y}$ at the end of the winter, then selection favors $\mathrm{x}$ above $\mathrm{y} . "$

\section{Population size change in GS}

A7.* Warm $\wedge \operatorname{dur}\left(\mathrm{E}_{1}\right)=$ long $\rightarrow \mathrm{N}\left(\mathrm{S}\right.$,end $\left.\left(\mathrm{E}_{1}\right)\right)=$ very_high $\wedge \mathrm{N}\left(\mathrm{G}\right.$, end $\left.\left(\mathrm{E}_{2}\right)\right)=$ mod_high

A8. ${ }^{*} \neg$ Warm $\wedge \neg\left(\operatorname{dur}\left(\mathrm{E}_{1}\right)=\right.$ long $) \rightarrow \mathrm{N}\left(\mathrm{S}\right.$, end $\left.\left(\mathrm{E}_{1}\right)\right)=$ medium $\wedge \mathrm{N}\left(\mathrm{G}\right.$, end $\left.\left(\mathrm{E}_{2}\right)\right)=$ mod_low

A9.* Warm $\wedge \neg\left(\operatorname{dur}\left(\mathrm{E}_{1}\right)=\right.$ long $) \rightarrow \mathrm{N}\left(\mathrm{S}\right.$, end $\left.\left(\mathrm{E}_{1}\right)\right)=$ high $\wedge \mathrm{N}\left(\mathrm{G}\right.$,end $\left.\left(\mathrm{E}_{2}\right)\right)=$ medium

A10. $\neg$ Warm $\wedge \operatorname{dur}\left(E_{1}\right)=$ long $\rightarrow \mathrm{N}\left(\mathrm{S}\right.$,end $\left.\left(\mathrm{E}_{1}\right)\right)=$ high $\wedge \mathrm{N}\left(\mathrm{G}\right.$,end $\left.\left(\mathrm{E}_{2}\right)\right)=$ medium 
Theorems. The set of assumptions now implies selection preferences. Table 4. summarizes the results. Figure 2 gives a visual representation of population dynamics in the eight environmental patterns.

Table 4. The CA model's predictions for the eight environmental patterns

Low Variability

High Variability

\section{Coarse Grain Fine Grain Coarse Grain Fine Grain}

$\begin{array}{lllll}\begin{array}{c}\text { Low Dissimilarity } \\ \text { non-supportive } \\ \text { social climate }\end{array} & \text { 1. Cricket } & \text { 2. Cricket } & \text { 3. Ant } & \text { 4. Ant } \\ \begin{array}{c}\text { High Dissimilarity } \\ \text { supportive } \\ \text { social climate }\end{array} & \text { 5. Cricket } & \text { 6. Cricket } & \text { 7. Ant } & \text { 8. Cricket }\end{array}$

T1. Theorem. If environmental change is of low variability, then selection favors the Cricket population (Table 4, cells 1-2, 5-6).

$$
\operatorname{Var}(\text { low }) \rightarrow \text { Selection_favors(C,A) }
$$

The La Fontaine tale says that the Ant fares better if winter is long. The winter can be long in absolute terms, and it can be long relative to the summer. Coarse environmental grain means long winter in absolute terms. High variability represents long winter in relative terms. If the two combines, then the Cricket fails (T2, Figure 2.5-6).

T2. Theorem. If environmental change is of high variability and the change is coarse grained, then selection favors the Ant population (Table 4, cells 3 and 7).

$\operatorname{Var}($ high $) \wedge$ Grain(coarse) $\rightarrow$ Selection_favors(A,C) 
Two cases are left. If variability is high and grain is fine, then the winter is not shorter than the summer, causing problems to the Cricket. However, if environmental grain is fine, then the absolute length of winter is short, what might give some chance for the Cricket population (Figure 1). Then, selection's preference depends on the social climate. In case of high environmental dissimilarity, the "warm" summer raises the Cricket population sufficiently big to counterbalance winter losses (T3, Figure 2.8). Otherwise, the Ant population is favored (T4, Figure 2.7).

T3. Theorem. If environmental change is of high variability and high dissimilarity and the change is fine grained, then selection favors the Cricket population (Table 4, cell 8).

$\operatorname{Var}($ high $) \wedge \operatorname{Diss}($ high $) \wedge$ Grain(fine $) \rightarrow$ Selection_favors $($ C,A $)$

T4. Theorem. If environmental change is of high variability and low dissimilarity and the change is fine grained, then selection favors the Ant population (Table 4, cell 4).

$\operatorname{Var}($ high $) \wedge \operatorname{Diss}($ low $) \wedge$ Grain(fine $) \rightarrow$ Selection_favors(A,C)

Hard times are over. No more formal theory building is necessary. Now, the task is to operate the "switch", that is, to fine tune the CA machinery by specific boundary conditions and to deduce the conclusions of GS and RK. Again, the emphasis is on the sociological insights: What are the differences between the three addressed population theories and how do these differences transform into model specifications? Let's begin with OE's niche theory (GS). I follow Hannan's and Freeman's (1989) description and denotations. 
Figure 2. Population growth and decline during the two seasons

The size of the Cricket population

- - - - _ - The size of the Ant population

\section{Low environmental variability}

1. Coarse grain, high

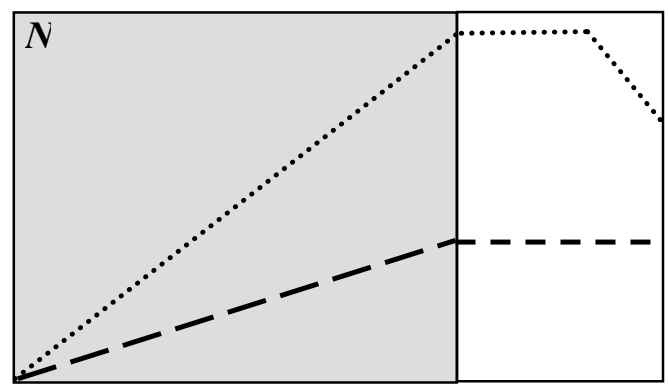

3. Fine grain, high dissimilarity

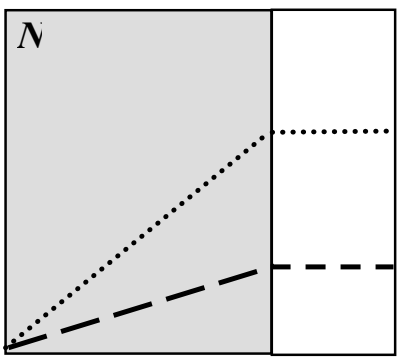

2. Coarse grain, low dissimilarity

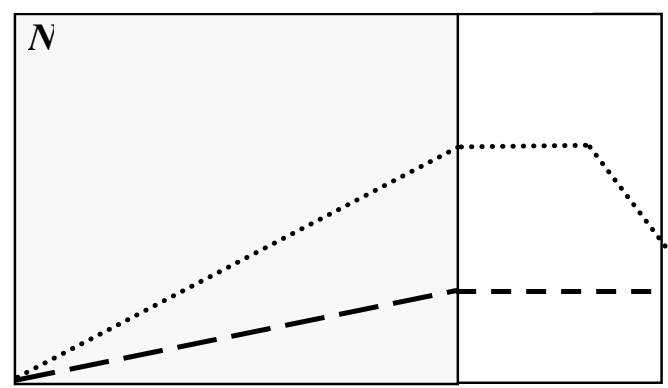

4. Fine grain, low dissimilarity

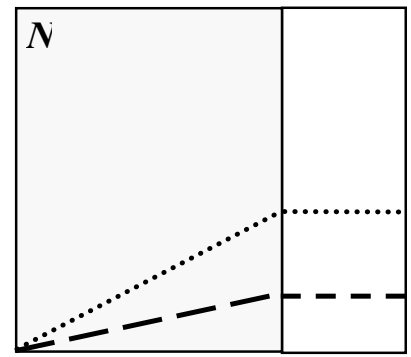


Figure 2 (cont.) Population growth and decline during the two seasons

The size of the Cricket population

- - - - _ . The size of the Ant population

\section{High environmental variability}

5. Coarse grain, high

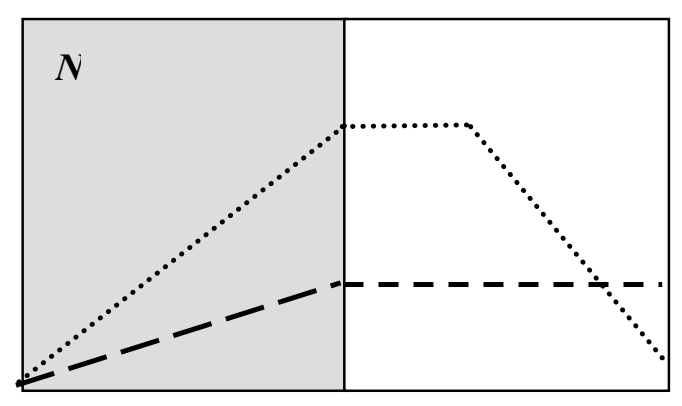

7. Fine grain, high dissimilarity

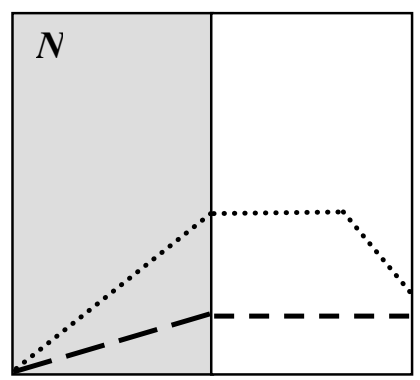

6. Coarse grain, low dissimilarity

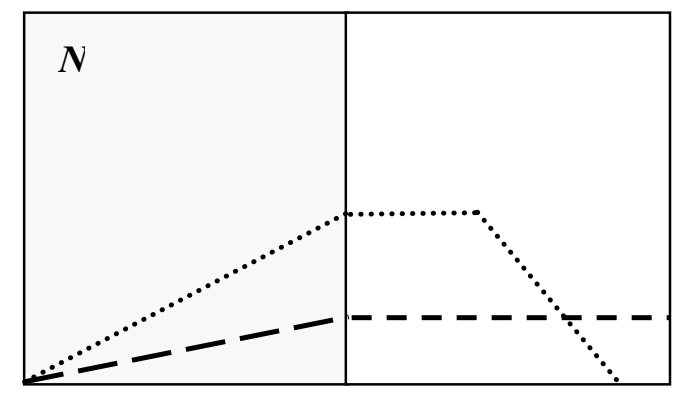

8. Fine grain, low dissimilarity

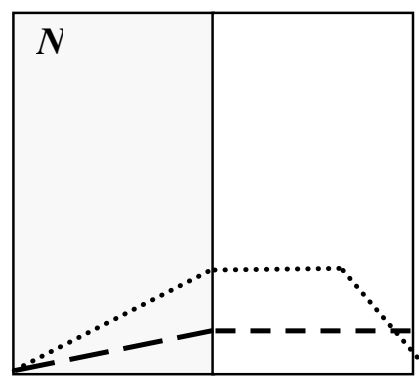




\section{Niche Strategies: Quality or Continuity?}

\section{Generalist and specialists in changing resource environments}

The niche of a population is given by the set of environmental conditions by which this population sustains (Levins 1968). Two resource environments $\left(E_{1}\right.$ and $\left.E_{2}\right)$ follow each other sequentially in GS. The eight patterns of environmental change are the same as in CA (Figure 1). But while the difference between the two altering resource states is quantitative in CA (presence and lack of external resource offer), it is qualitative in GS: $E_{1}$ and $E_{2}$ represent two different resource configurations. Organizations in the generalist population adapt to both $\mathrm{E}_{1}$ and $\mathrm{E}_{2}$ in the sense that they can operate under both conditions (broad niche). Specialists adapt only to $\mathrm{E}_{1}$ (narrow niche). The principle of allocation fixes that building up the ability to operate under both condition takes it toll: it lowers organizational fitness, so generalists have only a mediocre performance all the time. On the contrary, specialists perform very well under the narrow range of conditions $\left(E_{1}\right)$ they are specialized to. But, no resource utilization is possible for specialists when $E_{2}$ holds; then, they have to survive on reserves. Specialist organizations bet on the quality of operations, generalists bet on the continuity of operations.

The predictions of GS on selection preferences is as follows. In the four low variability cases $\mathrm{E}_{1}$ dominates (Figure 1.), so specialism is the favored strategy. But if environmental variability is high, then specialists face beneficial and hostile conditions for approximately the same duration. If the bad $\mathrm{E}_{2}$ periods are long, then specialists run out of their reserves and their population collapses; so, generalism is the favored strategy when high variability combines with coarse grain. However, if variability is high but grain is fine, then the $\mathrm{E}_{2}$ patches are not deadly long for specialists. Then, selection's preference depends on the dissimilarity between the two environments. The bigger the dissimilarity, the broader generalist niche is required to adapt both to $\mathrm{E}_{1}$ and $\mathrm{E}_{2}$, and this implies lower fitness (principle of allocation). Therefore, high variability and fine grain combined with high dissimilarity is better for specialists, while the same setting with low dissimilarity is better for generalists. The next step is to show that the CA model implies these predictions as theorems, provided that some GS conform modifications are performed.

\section{Modifications}

Environmental change has the same "good times - bad times" character for specialists in GS as it has for both the Cricket and Ant in CA. The specialist population behave exactly like the Cricket: it flourishes when $\mathrm{E}_{1}$ (summer) holds. $\mathrm{E}_{2}$ is inaccesible, so practically resourceless for specialists. $\mathrm{E}_{2}$ is not resourceless for generalists. However, the costs of 
adaptation to both environmental conditions (lower fitness), slows down the growth of the generalist population. Note the analogy with the Ant population's damped growth in CA because of reserve accumulation. The generalist and the Ant populations have a kind of life insurance against environmental change, where the policy cost comes in the form of slower growth. The generalists maintain multiple operational routines (Nelson and Winter 1982), one for $E_{1}$, and another for $E_{2}$, to buffer against qualitative resource change. The Ant population buffers out the disappearence of resource by heaping up reserves.

Replace the Cricket (C) for specialist (S), and the Ant (A) for generalist (G) in all pertaining formulae (Tables 2-3). Similarly, replace summer (S) and winter (W) for $\mathrm{E}_{1}$ and $\mathrm{E}_{2}$, respectively. Up till this point, only denotational modifications were made. The only non-denotational modification concerns the fact that the generalist population can grow in both environmental patches $\left(\mathrm{E}_{1}\right.$ and $\left.\mathrm{E}_{2}\right)$, while its analogue in $\mathrm{CA}$, the Ant, can grow only in the first patch (summer). At the level of the machinery, this means that the generalist population reaches its maximal size only at the end of the second patch $\left(E_{2}\right)$, instead of the first one. This modification affects formulae A7-10; their GS versions are denoted as A7*A10* (Table 3). The formal proofs indicate that this model change does not effect the theorems: exactly the same selection preferences follow from the modified premise, but now for specialists and generalists. Let's perform the Cricket $\sim$ Specialist, and Ant Generalist replacement in Table 4. The obtaining Table 5 reiterates niche theory's predictions described in the previous paragraph (see also Péli 1997, pp. 24).

Table 5. The GS model's predictions for the eight environmental patterns

\section{Low Variability}

\section{Coarse Grain}

Low dissimilarity 1 . Specialists

High Dissimilarity 5. Specialists

\section{Fine Grain}

2. Specialists

3. Generalists

4. Generalists

\section{Coarse Grain Fine Grain}

\section{High Variability}

6. Specialists 7. Generalists

8. Specialists 


\section{Propagation Strategies: Quality or Quantity?}

\section{$K$ - and $r$-strategies (RK)}

$K$ - and $r$-strategist organizational populations have been introduced by a biological analogy in OE (Brittain and Freeman 1980, Hannan and Freeman 1989). $K$ and $r$ denote, respectively, the carrying capacity and the intrinsic growth rate the in bioecology. Species that follow $r$-strategy produce several offspring. This strategy “... leads to many reproductive events with fixed energy and material resources spread thinly over attempts. Because the investment in each reproductive event is small, the life chances of each offspring are poor. However, populations using this strategy grow rapidly under favorable conditions." On the other hand, $K$-strategy “... makes a small number of reproductive events and invests much energy and time in each one. Individual offspring have relatively good life chances under this strategy" (Hannan and Freeman 1989, pp. 118). K-strategist biological populations have competitive advantages over $r$-strategists when free resources disappear, i.e., the carrying capacity of the location has been hit. Hannan and Freeman apply these two ways of propagation to organizational strategies: $r$-strategists' analogues are first mover organizations with the ability to exploit new opportunities quickly due to easy assembling; $K$-strategists' analogues go for efficient production. In the RK model, the tradeoff stands between the quality and the quantity of population membership. What serves better long the run success: having less but skilled organizations or more easy-to-assemble but poorly competing organizations?

The relative success of the two strategies depends on the stability of environmental conditions. The $r$-strategists flourish in the short run, while $K$-strategists' thorough investments yield their returns in the long run. Consequently, slow environmental change or stable conditions (long environmental patches) are favorable for $K$-strategists.

Environmental patches are short when change is rapid. Then, resource conditions may alter before $K$-strategists could overcome, and $r$-strategists may fare better. The description on $r$ strategists clearly fits to the Cricket population. The Ant population can also be seen as $K$ strategist: now those are not reserves, but the production skills that $K$-strategists build up.

A difference between $\mathrm{CA}$ and $\mathrm{RK}$ is that while $\mathrm{CA}$ is about two environmental states (one with and one without resources), there is a permanent environmental resource supply in RK. What constitutes then the two altering environmental patches? Recall that the growing populations bound all free resource after a certain period of time in RK. The time point when the carrying capacity is met divides the time scale into two phases: to an initial period with free resource $\left(\mathrm{p}_{1}\right)$, and to a subsequent period without free resource $\left(\mathrm{p}_{2}\right)$. This yields a two-step RK model, similar to CA. The change in resource offer is quantitative in both settings, but with a substantial difference. Resource availability only depends on the 
environment in CA. Resource offer is abundant or scarce in absolute terms, without respect to the presence of the populating organizations. However in RK, resource availability also depends on the level of resource consumption.

There is no resource competition between $r$ - and $K$-strategists in the first phase $\left(\mathrm{p}_{1}\right)$. $K$-strategists invest in skill development and these learning efforts set back their population's growth. Meanwhile, the $r$-strategist population has a free ride growth. In the second phase $\left(\mathrm{p}_{2}\right)$, organizations can only take additional resources from each other.

Resource competition becomes strong, and the population with better competitive skills outperforms the other in the long run. From a selection point of view, the crucial issue is if there is a "long run" at all: Are environmental conditions stable enough to let the $K$ strategists to overcome? OE's prediction is that slow environmental change favors $K$ strategy, while rapid change favors $r$-strategy (Hannan and Freeman 1989, pp. 118-120). The next step is to derive these conclusions by performing some RK-conform modifications on CA.

Table 6. The RK model's predictions for the eight environmental patterns

\section{Fine Grain}

slow change
Coarse Grain
rapid change

rapid change

1. $r$-strat.

Low Dissimilarity

non-supportive

social climate

High Dissimilarity 5. $r$-strat.

supportive

social climate

High Variability
Fine Grain

slow change 


\section{Modifications}

Again, I begin with the denotational modifications. The RK analogue of the Cricket is the $r$ strategist population in the sense that both have a rapid initial growth, and both face hard times when resource availability becomes poor. The $K$-strategist population is the analogue of the Ant in the sense that they both sacrifice short term advantages for better performance under resource scarcity. Denote $r$ - and $K$-strategists by $\mathrm{r}$ and $\mathrm{K}$, respectively. Replace $\mathrm{C}$ with $\mathrm{r}$, and replace A with $\mathrm{K}$. Change also summer (S) for $\mathrm{p}_{1}$ and winter $(\mathrm{W})$ for $\mathrm{p}_{2}$ in all pertaining formulae (Tables 2-3). The obtaining selection preferences in RK are listed in Table 6.

Recall that Hannan and Freeman (1989) claim that selection favors $K$-strategy if environmental change is slow, and that it favors $r$-strategy when change is rapid. Grain stands for the speed of environmental change in the given model characterization (Figure 1). Thus, the expectation is that coarse grained change favors $K$-strategists, while fine grained change favors $r$-strategists. However, Table 6 seems to show completely different selection patterns. The picture gets clearer if two additional assumptions are added to RK that are conform to Hannan's and Freeman's description (1989, pp. 118-123).

First, RK is about opening markets with a set of new, impressive opportunities (due to technological or market structure change) that attracts new entrants. In CA model terms, this means supporting social climate that facilitates rapid initial organizational growth. As supporting social climate corresponds to high environmental dissimilarity (cf. part 4), the upper line of Table 6 with low dissimilarity does not apply (cells 1-4).

Second, the carrying capacity of the new market is usually not extremely high. In case of extremely high resource offer (limitless demand, for example), organizations can sustain without skill development, and with low quality products even in the long run. Such cases may occur occasionally, but then the whole $r-K$ tradeoff is immaterial. An extremely high carrying capacity implies a very long initial period with free resource $\left(\mathrm{p}_{1}\right)$. Why? Because the supporting social climate allows for rapid resource absorption (easy growth) in $\mathrm{p}_{1}$. If $\mathrm{p}_{1}$ is still very long in spite of rapid resource absorption, then the environmental resource offer must be extremely high (extremely high carrying capacity). The $\mathrm{p}_{1}$ period is the longest, if coarse grain combines with low environmental variability (Figure 1). Thus, excluding extremely high carrying capacities excludes cells 1 and 5 in Table 6.

Three cases are left, cells 6-8, printed in bold in Table 6. In these cells, coarse grained environmental change favors $K$-strategists, and fine grained change favors $r$-strategists, as Hannan's and Freeman's argument implies. That is, applying two OE conform restrictions, the obtaining model yields the expected predictions. 
Note finally, that the formal machinery is not sensitive to the trajectories as to how populations reach their maximal size by the end of the first patch. The way how Figure 2 depicts initial growth is only one of several possibilities.

\section{The excluded cases}

The excluded environmental conditions (not supporting social climate in new markets, extra high carrying capacity) may occur sometimes in reality. Can we see the broader RK model, the one without the two added constraints, as a potential generalization of the original theory? Is there a reasonable sociological interpretation for RK's predictions for the excluded cases (cells 1-5, Table 6)?

In the four low dissimilarity cases (cells 1-4, Table 6), the social climate is not supportive. The only difference between the low and high dissimilarity cells occurs when high variability combines with fine grain. Then, $K$-strategy is favored in case of supportive social climate (high dissimilarity), and $r$-strategy is favored otherwise. A not supporting social climate implies slower growth for both populations during $\mathrm{p}_{1}$. However, a cool social climate, being an external environmental feature, effects much more the rapid growth oriented $r$-strategists than the $K$-strategists. Skill development, the strength of $K$-strategists, depends rather on internal organizational aspects. This explains why $K$-strategy is favored instead of $r$-strategists in cell 4 (Table 6). It is only environmental variability that decides selection preferences in cells 1-4. Low variability, when $\mathrm{p}_{1}$ with free resource is much longer than $\mathrm{p}_{2}$ without free resource (Figure 1), favors $r$-strategists. The second phase, $\mathrm{p}_{2}$, is just too short relative to $\mathrm{p}_{1}$ for $K$-strategists to outcompete $r$-strategists. But, when environmental variability is high, then $\mathrm{p}_{1}$ and $\mathrm{p}_{2}$ are of about the same duration and this allows sufficient time for $K$-strategists to overcome.

In the excluded case of extra high carrying capacity, when high environmental dissimilarity combines with low variability and coarse grain, $r$-strategists are favored by selection (cell 5, Table 6 ). Then, the long initial period $\left(\mathrm{p}_{1}\right)$ with supportive social climate allows for a very big $r$-strategist population that can keep its size advantage over $K$ strategists in the relatively short competitive $\mathrm{p}_{2}$ period. Some new East-European markets that have been opened after the fall of the Iron Curtain exemplified the case of very long initial period (Czechia, Hungary and Poland). Customers' demand, carrying capacity, is very high due to several decades of shortage economy. As a consequence, a big number of $r$ strategist firms with low quality products could be seen there for a couple of years without facing serious resource competition. 
$\begin{array}{lll}\text { CA GS } & \text { RK }\end{array}$

\begin{tabular}{lccc}
\multicolumn{1}{c}{ Phases } & summer, winter & $\mathrm{E}_{1}$ and $\mathrm{E}_{2}$ & $\begin{array}{c}\text { before / after reaching } \\
\text { the carrying capacity }\end{array}$ \\
$\begin{array}{c}\text { Difference in } \\
\text { resource } \\
\text { availability }\end{array}$ & quantitative & qualitative & quantitative \\
$\begin{array}{c}\text { Resource in the } \\
\text { second phase }\end{array}$ & not present & present & present \\
$\begin{array}{c}\text { Free resource in } \\
\text { the second phase }\end{array}$ & not present & present & not present \\
$\begin{array}{l}\text { Remedy against } \\
\text { resource scarcity }\end{array}$ & $\begin{array}{c}\text { buffering } \\
\text { (Ant) }\end{array}$ & $\begin{array}{c}\text { adaptation } \\
\text { (Generalists) }\end{array}$ & $\begin{array}{c}\text { competitive skills } \\
(K \text {-strategists })\end{array}$
\end{tabular}

\section{Discussion}

The paper specified a new population dynamics model (CA) for punctual and quantitative environmental resource change. CA shows some substantial similarities to two well established population dynamics models of organizational ecology (GS and RK). For example, organizational populations face strategic tradeoffs between short term benefits (growth) and long term safety due to reserves (CA), or alternative skills (GS), or high quality skills (RK). The proposed logical model highlights the similarities (reflected by the common model core) as well as the differences (reflected by the specific boundary conditions) in the three population theories. Analogies in population behavior come upfront. Table 7 lists some of the differences and analogies between the three component models. The paper demonstrated that the predictions of CA, GS and RK obtain as theorems as the specific assumptions of the three population models are applied to core machinery.

Resource change. The temporal patterns of environmental change are the same in the three models. But environmental conditions differ in CA, GS and RK in another respect: the 
composition of environmental resource offer alters in different ways. In case of qualitative resource change, a non-zero flow of different resource combinations sustains in both phases; then OE's niche theory applies. In case of quantitative resource change, high and low seasons alter; then the Cricket and Ant tradeoff applies.

Qualitative resource change also can be expressed with quantities. Consider the set of all possible sorts of environmental resource, and denote the cardinality of this set with $k$. Then, all resource configurations can be expressed with a $k$-vector, where the resource sorts missing from the configuration are represented with zero components. The disappearence of all resource (quantitative change to winter in CA) corresponds to a zero vector. As quantitative resource change can be reduced to a special case of qualitative change, the Cricket and Ant model might be seen as a special case of OE's niche theory. In fact, arguments about niche width effects and reserve effects on survival come together in the argumentation of niche theory. Generalist organizations "hold some capacity in reserve" to ensure reliability of performance when conditions change, while "some of the efficiency resulting from specialism derives from lower requirements for maintaining excess capacity" (Hannan and Freeman 1989, pp. 106). If the environmental resource flow is not zero, then generalists' reserves need not play a role in GS (see Péli 1997). But even then, specialists' short survival period in $\mathrm{E}_{2}$ is due to their short supply of accumulated resource. As qualitative and quantitative resource change can come together, organizational populations may face the Cricket-Ant and the Specialist-Generalist tradeoffs at the same time. GS and CA can be seen as the two marginal cases, when only the composition and the magnitude of resource supply counts, respectively.

Another way of quantitative resource change comes from the presence of a finite, non-zero carrying capacity; then RK applies. In CA, it is the magnitude of environmental resource inflow that changes. In RK, expanding organizational populations consume an increasing percentage of the external resource flow. What organizations perceive in both models is that resource availability gets worse with time. In CA, organizations may accumulate reserves. In RK, competitive $K$-strategist organizations take the resource share of their neighbors. An alternative interpretation of $K$-strategy is that skill elaboration is not about becoming a tough competitor but about the more efficient exploitation of the firm's own assets. This yields a pacifist version of RK, in which K-strategists "produce" free resource by freeing up a part of their previously bound reserves via efficient production.

The populations in the three models may of course interact. But surprisingly, all three models work also without assuming interaction between populations. In CA and GS, organizations fail because of problems of finding or absorbing resource. In RK, organizations disband because others diminish their resource share; but even then the parties need not interact or even know about each other, i.e., resource competition can be diffuse. In CA, the Cricket and the Ant need not even populate the same market; the same 
selection outcomes obtain if the two are located at separate markets characterized with similar resource conditions. The same applies to GS. However in RK, a rearrangement of bound resource between populations takes places in the second phase $\left(\mathrm{p}_{2}\right)$, involving that $r$ and $K$-strategists are located at the same market.

Theory unification? Physics provides nice examples when different parts of theories are built into a common conceptual framework. Maybe the best known example is Einstein's general relativity theory (1915) where the extra gain in explanatory power stems from the unification of inertial mass (that reflects bodies' resistance against motion state change) and gravitational mass (proportional to the gravitational force between bodies) into one notion. An earlier example is Maxwell's electromagnetic theory (1899) that shows that electric and magnetic effects can be seen as two facets of one phenomenon: it depends on the motion state of the observer (still or moving relative to the charged bodies), if $\mathrm{s} / \mathrm{he}$ detects electronic or magnetic field.

Can the work performed in this paper be seen as theory unification? Theory unification involves a common conceptual model that gives back the component theories as special cases of the general theory. The proposed logical model fulfils this requirement: adding CA, GS and RK specific constraints to the model core, the respective theories obtain. However, in the referred physical theory unifications, different key objects of the particular theories were unified into one in the general theory (inertial and gravitational mass, electronic and magnetic effects). In the presented logical formalization, the key objects in the three component theories do not merge into one in the context of the general model. For example, it is not the case that the same population can bee seen as Ant, Generalist and $K$-strategists as the focus of the analysis is placed on the respective quantitative, qualitative and carrying capacity related aspects of resource supply. What the paper demonstrates is that three population dynamics approaches can be represented with one model machinery. This is less than theory unification. However, it may be a definite step towards it.

\section{References}

Barron, David N., Elisabeth West and Michael T. Hannan .1994. "A Time to Grow and a Time to Die: Growth and Mortality of Credit Unions in New York, 1914-1990." American Journal of Sociology. 100:381-421.

Barwise, Jon and J. Etchemendy. 1990. The Language of First-Order Logic. CSLI 23. Stanford University: Chicago University Press. 
Brittain, Jack and John Freeman. 1980. "Organizational Proliferation and Density Dependent Selection." Pp. 291-338 in Organizational Life Cycles, edited by J. R. Kimberly and R. H. Miles. San Francisco: Jossey-Bass.

Bruggeman, Jeroen. 1997. "Niche width theory reappraised." Journal of Mathematical Sociology, 22:201-220.

Carroll, Glenn R. 1985. "Concentration and Specialization: Dynamics of Niche Width in Populations of Organizations.” American Journal of Sociology, 90: 1262-1283.

Carroll, Glenn R. and Michael T. Hannan. 1989. "Density Delay in the Evolution of Organizational Populations: A Model and Five Empirical Tests." Administrative Science Quarterly. 34:411-430.

Freeman, John and Michael T. Hannan. 1983. "Niche Width and the Dynamics of Organizational Populations." American Journal of Sociology. 88: 1116-1145.

Gamut, L. T. F. 1991. Logic, Language and Meaning. Chicago: Chicago University Press.

Hannan, Michael T. 1998. "Rethinking Age Dependence in Organizational Mortality: Logical Formalizations.” America Journal of Sociology 104: 85-123.

Hannan, Michael T. and John Freeman. 1977. "The Population Ecology of Organizations." American Journal of Sociology. 82: 929-964.

Hannan, Michael T. and John Freeman. 1989. Organizational Ecology. Cambridge. Massachusetts, London: Harvard University Press.

Hannan, Michael T. and Glenn R. Carroll. 1992. Dynamics of Organizational Populations. Density, Legitimation and Competition. Oxford University Press. New York, Oxford.

Kamps, Jaap and László Pólos. 1999. "Reducing Uncertainty: A Formal Theory of Organizations in Action." American Journal of Sociology. 104:1776-1812.

La Fontaine, Jean de. 1966. Fables. Paris. Garnier-Flammarion.

Levins, Richard. 1968. Evolution in Changing Environments. Princeton New Jersey: Princeton University Press. 
Nelson, Richard R. and Sidney G. Winter. 1982. An Evolutionary Theory of Economic Change. Cambridge, Massachusets. Harvard University Press.

Péli, Gábor. 1997. “The Niche Hiker's Guide to Population Ecology: A Logical Reconstruction of Organization Ecology's Niche Theory." Sociological Methodology. 27:146.

Péli, Gábor and Michael Masuch. 1997. "The Logic of Propagation Strategies: Axiomatizing a Fragment of Organizational Ecology in First-Order Logic." Organization Science. 8:310-331.

Péli, Gábor, László Pólos and Michael T. Hannan. 2000. "Back to Inertia: Theorethical Implications of Alternative Styles of Logical Formalization." Sociological Theory (forthcoming)

Szabó, Miklós. 1981. "Az aranytojást tojó tyúk történetéhez." (Some Additions to the Golden Egg Laying Hen Story.) In Hungarian. Medvetánc 1981/1.

Tompson, James D. 1967. Organizations in Action: Social Science Bases of Administrative Theory. New York. McGraw-Hill. 